\title{
Treating opioid use disorders in drug court: Participants' views on using medication- assisted treatments (MATs) to support recovery
}

John R. Gallagher, PhD, LSW, LCAC (corresponding author) Associate Professor, School of Social Work Indiana University South Bend (IUSB)

Wiekamp Hall, 2221 1800 Mishawaka Avenue, PO Box 7111

South Bend, IN 46634-7111

(574) 514-1813

johngall@iupui.edu

Elizabeth A. Wahler, PhD

Associate Dean/Associate Professor, School of Social Work

Indiana University Purdue University Indianapolis (IUPUI)

Raychel M. Minasian, MSW

Graduate Research Assistant, School of Social Work

Indiana University South Bend (IUSB)

Abigail Edwards, MSW

Graduate Research Assistant, School of Social Work

Indiana University South Bend (IUSB)

This is the author's manuscript of the article published in final edited form as:

Gallagher, J. R., Wahler, E. A., Minasian, R. M., \& Edwards, A. (2019). Treating Opioid Use Disorders in Drug Court:

Participants' Views on Using Medication-Assisted Treatments (MATs) to Support Recovery. International Criminal Justice Review, 29(3), 249-261. https://doi.org/10.1177/1057567719846227 


\begin{abstract}
Drug courts began in 1989 in Miami-Dade County, Florida, U.S.A. Due to their success in treating substance use disorders and reducing criminal recidivism, they have expanded globally and are currently operating in countries such as Australia, Canada, and Scotland, to name a few. Drug courts can be a key intervention in addressing the opioid epidemic. This is the first known qualitative study to ask drug court participants $(\mathrm{n}=38)$ who have an opioid use disorder questions related to their lived experiences in drug court, as well as direct questions related to the use of medication assisted treatments (MATs) in drug court. Overall, drug court participants felt that MATs were helpful for treating their opioid use disorders; however, some participants reported using other drugs while on MATs and they viewed their recovery through a harm reduction lens. Additionally, participants emphasized the importance of using MATs in combination with counseling that used cognitive and behavioral therapies. Implications for drug court practice and future research are discussed.
\end{abstract}

Keywords: drug court, harm reduction, medication-assisted treatment, opioid use disorder, qualitative research 


\section{Introduction}

The United States of America (U.S.) is currently experiencing an opioid epidemic that is having devastating consequences on individuals and families, the healthcare system, criminal justice system, and overall economic wellbeing of the country. In 2015, more than 33,000 Americans died prematurely from an opioid-related drug overdose (The Council of Economic Advisers, 2017). Overall, the estimated economic impact of the opioid epidemic on the U.S. is \$504 billion dollars; this estimate includes all consequences of the opioid epidemic, including overdose deaths and other expenses such as increased criminal justice costs for the policing, arresting, and incarceration of individuals who possess or sell illicit opioids (The Council of Economic Advisors, 2017).

While the negative consequences of the opioid epidemic seemed to have appeared rapidly, the epidemic is actually an unintended consequence of U.S. medical trends that began approximately three decades ago (U.S. Department of Health and Human Services [HHS], 2018). During the 1990s, prescriptions for opioids began to increase and become one of the standard, first options for treating pain. Recent data show that U.S. pharmacies dispensed 214,881,622 opioid prescriptions in 2016, resulting in $66.5 \%$ of Americans receiving a prescription opioid that year (Center for Disease Control and Prevention, 2017). In 2017, opioid addiction was declared a national public health emergency (HHS, 2018).

As the number of opioid prescriptions has climbed, the rates of opioid use disorders have increased, too. Although not a process that happens for everyone, prescription opioid use can progress to illicit drug use via the development of tolerance and withdrawal, two primary symptoms of a substance use disorder (Substance Abuse and Mental Health Services Administration [SAMHSA], 2018). For instance, an individual who has been taking an opioid 
(e.g. Vicodin) as prescribed may develop a physiological tolerance to the drug and therefore need more of the drug to get the desired effect. They may take more of their medication than prescribed and therefore not have enough to last an entire month, leading to withdrawal when they no longer have the medication. To avoid powerful and overwhelming withdrawal symptoms, the individual may turn to illicit behavior such as buying heroin or fentanyl illegally. This pattern is common, and many illicit opioid users originally began their use with prescription opioids (Center for Disease Control and Prevention, 2017). Thus, if effective treatment is not an option, many opioid dependent individuals may resort to criminal behavior to sustain their substance dependence. Besides the resultant increased risk of criminal justice involvement, users of illicit opioids are also at higher risk of overdose and death due to the powerful nature of these drugs and the difficulty in estimating their strength when buying illegally; U.S. opioid-related overdoses are currently at an all-time high (Rudd, Seth, David, \& Scholl, 2016).

The U.S. criminal justice system is overburdened with people who have been convicted of drug-related crimes, yet lacks the ability to effectively treat substance use disorders. Nearly half a million Americans were incarcerated for drug-related offenses in 2016 (The Sentencing Project, 2018). At the federal level, nearly half of the prison population is comprised by people who had drug-related convictions. However, only about $15 \%$ of inmates who have drug use disorders receive needed treatment (Bronson, Stroop, Zimmer, \& Berzofsky, 2017). The opioid epidemic has contributed to these rates, and approximately $22 \%$ of incarcerated individuals report regularly using heroin or other opiates prior to their incarceration (Bronson et al., 2017). Medication assisted treatments (MATs) are a harm-reduction approach taken to reduce the risk of morbidity, mortality or other negative consequences of chronic opioid misuse. With MATs, opioid agonist medications are prescribed and closely monitored to control the level of 
medication ingested, thereby reducing drug-seeking behaviors and intense highs and withdrawal periods associated with illicit drug use (National Institute on Drug Abuse [NIDA], 2016; SAMHSA, 2018; Volkow, 2014). Prescribed opioid agonists have been found to decrease risk of mortality, illicit opioid use, infectious disease transmission, and criminal activity as well as increase employment for people who are dependent on opioids (NIDA, 2016). However, these medications are not always used or encouraged by the criminal justice system due to an abstinence-based philosophy of drug treatment or concerns about potential drug diversion (Friedmann et al., 2012).

\section{Use of the Criminal Justice System for Treatment}

The criminal justice system can play a key role in addressing the opioid epidemic, particularly by providing enhanced treatment and recovery services to individuals coming in contact with the system. This addresses one of the U.S. Department of Health and Human Service's (HHS) priorities for addressing the opioid epidemic, enhanced treatment and recovery services for people with opioid use disorders (HHS, 2017). Drug courts are one such option for ensuring that treatment and recovery-related services are combined with the criminal justice system, both in the U.S. and internationally.

Drug courts are specialized programs that target offenders with substance use disorders or drug-related crimes. These programs are problem-solving courts that provide screening and assessment, treatment and recovery services, monitoring, and progressive incentives/sanctions to provide support for recovery. Drug courts have seen tremendous growth both inside and outside the U.S. since beginning in 1989, and there are now approximately 3,100 drug courts in the U.S. (U.S. Department of Justice, 2018). Drug courts have expanded globally, operating in countries such as Australia, Brazil, Canada, England, Ireland, Jamaica, and Scotland (Holst, 2010). 
Three decades of evidence have shown that drug courts appear to be more effective than traditional criminal justice interventions (such as probation) at reducing criminal recidivism rates for individuals who have substance use disorders and arrests for nonviolent offenses. The evidence that drug court participants recidivated at lower rates than comparison and control groups is highlighted in quasi-experimental studies of single drug courts (Brown, 2011; Gallagher et al., 2015), rigorous evaluations that used experimental research designs (Gottfredson \& Exum, 2002; Gottfredson, Najaka, \& Kearley, 2003) and meta-analytic reviews (Mitchell, Wilson, Eggers, \& MacKenzie, 2012; Shaffer, 2011a). More recent studies have moved beyond quantitative outcome studies to focus qualitatively and primarily on understanding barriers to and improving outcomes for certain populations that drug courts serve, such as women or African Americans (see Gallagher \& Nordberg, 2017; Gallagher \& Wahler, 2018; Vandermause, Severtsen, \& Roll, 2013). However, no known studies have examined the lived experiences of drug court participants who have opioid use disorders.

\section{Drug Courts and Treatment of Opioid Use Disorders}

Although designed to ensure offenders receive needed drug treatment, U.S. drug courts have been criticized for sometimes failing at this goal (Matsuow et al., 2013; Mehta \& Møllmann, 2017). A recent survey of drug courts in the U.S. found that nearly all drug courts surveyed (98\%) served participants who had opioid use disorders, yet only $56 \%$ offered or allowed MATs (Matsuow et al., 2013). Observed barriers to using MATs in drug court included cost, but also opposition from key stakeholders, such as the drug court judge and prosecutor (Matusow et al., 2013). In a similar study, Friedmann and colleagues (2012) also found that MATs were infrequently allowed or used in criminal justice settings, such as drug courts, probation, and parole. When used, MATs were most frequently used by pregnant women who 
had opioid use disorders and were in opioid withdrawal. This is most likely because of federal and international guidelines urging the use of MATs for opioid-dependent pregnant women due to the associated reduction in risk and harm to the fetus (American College of Obstetricians and Gynecologists, 2017). Friedmann et al. (2012) found that non-pregnant drug court participants or individuals re-entering the community after incarceration were commonly denied or did not have access to MATs, despite the benefit these populations could also gain from these treatments.

The lack of MATs used in some drug courts may contribute to participants who have opioid use disorders having poorer outcomes, such as lower graduation rates or higher relapse or recidivism rates. A recent study by Gallagher and colleagues (2018b) found that participants who identified opioids as the primary drug used were $80 \%$ less likely to graduate, as compared to drug court participants who identified non-opioids as their primary drug. Only $30 \%$ of participants who primarily used opioids successfully completed drug court, whereas nearly $70 \%$ of participants successfully completed who primarily used non-opioids. Therefore, developing an in-depth understanding of drug courts and how they serve participants who have opioid use disorders is a priority. The current study is the first known qualitative study to explore the lived experience of drug court participants who have an opioid use disorder and their opinions about the use of MATs in drug court.

\section{Methodology}

\section{Qualitative Research Design and Sampling}

This study was approved by the Institutional Review Board (IRB) at the authors' university. The population of interest was drug court participants who have an opioid use disorder, so all participants were recruited from this group and self-identified as having been diagnosed. Participants were asked the questions in Table 1, focused on their perceptions of 
helpful aspects of drug court, how drug court could be more helpful, the benefits and challenges of using MATs, and whether drug court effectively uses MATs to treat people who have opioid use disorders. These questions were asked in focus groups, which are recommended when research participants have similar characteristics, such as being a member of drug court and having an opioid use disorder, and due to these similarities, may complement one another's experiences and add to an in-depth understanding of a particular phenomenon (Padgett, 2016;

Rubin \& Babbie, 2008).

Table 1 Focus Group Questions

1. Could you please describe what aspects of drug court are most helpful to you in treating your opioid use disorder?

2. Could you please describe how drug court could be more helpful to you in treating your opioid use disorder?

3. Could you please describe your thoughts and/or experiences on the benefits of using medication-assisted treatments to treat your opioid use disorder?

4. Could you please describe your thoughts and/or experiences on the challenges of using medication-assisted treatments to treat your opioid use disorder?

5. Could you please describe your thoughts and/or experiences on whether or not the drug court effectively utilizes medication-assisted treatments to treat participants who have opioid use disorders?

Research participants $(\mathrm{n}=38)$ were recruited in 2018 from one drug court located in a Midwestern state in the U.S. Two researchers attended six drug court hearings, which were held every Monday, to recruit research participants. The two researchers facilitated one focus group each Monday immediately after the hearing for consenting participants; therefore, they attended multiple drug court hearings to assure all who were eligible to participate in the research had an opportunity to do so. During the recruitment process, the researchers introduced themselves, described the research question and format of focus groups, highlighted that participation in the 
research was voluntary and confidential, and mentioned the inclusion criteria. Inclusion criteria were: (1) must be a current participant in the drug court; (2) must be able to comprehend, speak, and read English; (3) must be 18 years of age or older; and (4) must have an opioid use disorder. Individuals who met the inclusion criteria and volunteered to participate were scheduled for the focus group after their hearing. In order to promote convenience for the research participants, the focus groups were facilitated in the same building as the drug court, but on a different floor. Focus groups were facilitated in a private, closed conference room on a secure floor.

The focus groups were co-facilitated by two researchers and audio reordered. The focus groups were semi-structured; specifically, research participants were asked the five open-ended questions noted in Table 1 and probing questions were used to develop an in-depth understanding of their lived experiences in drug court. For example, the researchers used validating statements and probing questions such as, "It sounds like you benefited from using Suboxone (buprenorphine/naloxone). Could you please elaborate on the specific benefits you received by using the medication?" and "Balancing all the responsibilities of drug court with life seems to be a challenge. Could you please further describe the specific challenges you face?" Informed consent was received by all those who chose to participate in a focus group.

\section{Qualitative Data Analysis Plan}

The audio recordings of the focus groups were transcribed verbatim and uploaded to NVivo, a qualitative data analysis software. According to Miles, Huberman, and Saldana (2014), qualitative data analysis is theoretically driven; the data from this study were analyzed through a phenomenological lens. Phenomenological analysis explores the lived experiences of research participants, as well as the context of those experiences (Padgett, 2016). In this study, research participants were asked questions about their lived experiences in drug court and also 
encouraged to elaborate on how the experiences have impacted their lives, or perhaps the lives of others. The qualitative data analysis followed a four-step process (Miles et al., 2014; Rubin \& Babbie, 2008) and strategies, such as negative case analysis, were used to increase the rigor of the data analysis and validity of the findings (Padgett, 2016). First, to promote immersion in the data, the researchers read the transcriptions on four occasions over a two-week period. During this time, and consistent with phenomenological analysis, the lived experiences of drug court participants were continuously compared and contrasted to develop insight into the research question. Second, concept mapping was used to group data, identify codes, and provide a conceptual framework for the findings. Third, codes were grouped as themes, the number of research participants who contributed to each theme was quantified, and direct codes from the research participants were used to conceptualize each theme. Fourth, data that did not demonstrate consistent responses from the research participants were considered outliers and used for the negative case analysis.

Four strategies were used to increase the rigor of the data analysis and validity of the findings (Padgett, 2016). First, the data collection and analysis were completed by researchers from different disciplines (criminal justice, psychology, and social work) offering interdisciplinary triangulation. Second, peer debriefing allowed the researchers to share their preliminary codes and themes with colleagues who had expertise in qualitative research and seek their feedback on the logic of the data analysis and preliminary findings. Peer debriefing was completed via email and phone calls. Those involved in the peer debriefing process had access to all the transcripts, which were in anonymous format, meaning no names or other potential identifying information was on the transcripts. Third and fourth, negative case analyses and audit trails were completed for each theme, and the negative cases are presented in the findings 
section. The negative case analyses promote a balanced interpretation of the findings, and the audit trails provide an additional avenue to verify the findings.

\section{Findings}

Thirty-nine drug court participants met the inclusion criteria and 38 chose to participate, providing a response rate of $97 \%$. From May 2018 to July 2018, the researchers facilitated 6 focus groups. A brief demographic survey was given at the start of each focus group. The average age of focus group participants was 34 years old and the majority were male (63\% male, $\mathrm{n}=24 ; 37 \%$ female, $\mathrm{n}=14)$. Almost three-fourths of the sample were White $(71 \%$ White, $\mathrm{n}=$ 27; 29\% non-White, $\mathrm{n}=11$ ). The focus group questions (Table 1) were related to participants' thoughts and experiences in drug court and the use of MATs to support recovery. All $(100 \%, \mathrm{n}=$ 38) of the research participants had an opioid use disorder, and the majority $(58 \%, \mathrm{n}=22)$ had been on a MAT at some point in their life. Furthermore, $37 \%(n=14)$ of the research participants were on a MAT at the time of the focus group. For the participants who were on a MAT at the time of the focus group $(n=14), 50 \%(n=7)$ were on Suboxone (buprenorphine/naloxone), 29\% $(n=4)$ on Vivitrol (naltrexone), and 21\% $(n=3)$ on methadone. Throughout the analysis, two primary themes emerged from the data, as discussed below.

\section{The importance of combining MATs with cognitive and behavioral therapies}

Twenty-three of the 38 participants (61\%) shared thoughts, opinions, and lived experiences about their perceptions of the importance of combining MATs with psychosocial treatments such as cognitive and behavioral therapies. This theme is demonstrated through the quotes below. Overall, the majority of participants shared that one of the most helpful aspects of drug court in treating their opioid use disorders was participating in treatment. One participant, for instance, shared that whether or not you are on a MAT, cognitive or behavioral treatment is 
helpful in many areas of life, such as family relationships and employment. She also mentioned the importance of learning healthy coping skills, which tend to be taught through cognitive and behavioral therapies. Specifically, she said:

I'm in the women's matrix program at [name of treatment center] and it's a lot different than IOP [intensive outpatient program]. We talk about different life skills, how to cope with drugs, family life, work life, everything. Whether you are on a medication-assisted treatment or not, for us with heroin addictions, it's important for us to attend treatment each week.

Another participant discussed how opioid use negatively impacted his cognitions. The participant also noted the importance of combining MATs with other forms of treatment, specifically cognitive restructuring in his case. The participants shared:

It's good to get into treatment right away. It gives you at least a head start, you know? Trying to go through all of this with being sick and being out there on the streets is impossible. We need Suboxone or whatever else helps and treatment. Like I said, it gives you a head start. The counselors teach you how to think clear because when you're using drugs, you aren't thinking straight. Your mind changes completely, you know, and it gets your mind thinking, I can start life again.

The following participant shared a lived experience highlighting the importance of combining MATs with cognitive or behavioral treatment and recovery support groups. For some participants, MATs alone may not be enough to support ongoing recovery. The participants stated: 
You need to work a program. I need to work a program and that involves using a medication, Vivitrol, and going to counseling and meetings [recovery support groups]. Unless you're working a program, it's not going to work. Going to counseling, you know, working on your head stuff is what you need. Vivitrol isn't going to change your head stuff, your thinking about getting high.

Another participant candidly shared about his experience in psychosocial treatment, "We talk about relapse prevention and how to handle your emotions. Without that, I'd be in jail, getting high, or dead." One participant even mentioned attending psychosocial treatment more often than required by drug court. The participant said, "Although treatment wants you to do one a week, I take advantage of the support and go like four or so times a week." Other participants stated, "I think it's just obvious that you need treatment to go along with the medically assisted treatment" and combining MATs with psychosocial treatment results in, "improved mood, less cravings, just feeling better, and feeling more motivated."

Overall, the majority of participants felt that one of the most helpful aspects of drug court in treating their opioid use disorders was participating in psychosocial treatments. Participants, whether or not they were on a MAT, provided examples on how the use of cognitive (e.g. cognitive restructuring) and behavioral therapies (e.g. relapse prevention) supported their recovery, and they felt that MATs should be used in combination with these types of treatment. The negative case analysis revealed that only 3 (8\%) participants had experiences that conflicted with the theme. All three comments, however, were related to difficulties with balancing the demands of psychosocial treatment with other responsibilities, as compared to viewing these additional treatments as unhelpful. For example, a participant shared this lived experience: 
They [psychosocial treatment] don't understand a lot with all the stuff we have to do. They [psychosocial treatment] set it up to make sure, you know what I mean, that we are busy. With all the counseling we have to attend, it gets stressful and, ask everybody, it's a hell of a lot of stress to stay sober.

\section{The uneasy relationship between harm reduction and drug court programming}

Twenty of the 38 participants (53\%) shared thoughts, opinions, and lived experiences that contributed to the theme of an uneasy relationship between harm reduction and abstinence-based drug court programming, demonstrated through the quotes below. Overall, the majority of participants had favorable views toward harm reduction. In this case, harm reduction was conceptualized as reducing or abstaining from illicit opioid use, such as heroin, even if continuing to use other substances, and they viewed MATs as an important intervention in achieving this goal. The problem, however, is that the drug court in this study is guided by an abstinence-based_philosophy of treatment, consistent with the majority of U.S. drug courts. Therefore, participants who maintain abstinence from opioids but use other drugs can face sanctions from the drug court, which may include incarceration. Throughout the focus groups, multiple participants shared their observations that other drug court participants have abstained from opioids in drug court but began to use other substances. One participant, for instance, shared this observation:

One of the other downfalls is the shift in the drug of abuse, or drug of use. The switch from opiates over to methamphetamine. Stuff like that. That is one thing we've seen a lot more of, more positive drug tests with the medication-assisted treatment guys, especially positives for methamphetamine. 
For some of the participants, methamphetamine was most commonly identified as the drug used to replace opioids. It is unlikely that this phenomenon is due to a correlation between MATs and methamphetamine. A more plausible explanation is that methamphetamine was more readily available in the community; therefore, this increased the likelihood of methamphetamine use for those who were abstaining from opioids but wanted to use another drug. Regardless, there was a process of substituting opioids for other drugs for some participants. One participant discussed the cognitive process that may explain why some individuals on MATs use non-opioids, such as marijuana or cocaine. Specifically, the participant said:

There is a perception because heroin is so devastating, so fast, that even highly addicted people with very advanced substance use disorders know that, oh my god, I got to get off this. They take the med [medication-assisted treatment], they're off heroin and start thinking, alright, now I can do these other things. Alcohol was never my problem, or weed should be legalized anyway, or what's wrong with a little cocaine, it's not heroin.

The process of substituting one drug for another does not seem to be limited to stimulants, such as methamphetamine or cocaine, or marijuana. Some participants shared their lived experiences with using a MAT outside of medical supervision to maintain their recovery from heroin. In this instance, a participant shared the experiences of others using a non-prescribed MAT to prevent a relapse on heroin. The participant shared:

I have known people who were using heroin and they don't want to relapse on heroin, so they'll go find somebody who has Suboxone and they'll use their Suboxone instead of relapsing on heroin. They think that's a better alternative than going back and using heroin. 
The following participant shared that his drug of choice was heroin and, consistent with a harm reduction model of recovery, his goal was to maintain abstinence from his drug of choice. Furthermore, the participant gave an example of how others also see it as progress and offer praise when an individual maintains abstinence from heroin despite also using other substances. The participant stated:

They still kind of look down upon it, of course, because, you know, it's still you failed [positive drug test] for something, but they still do high-fives and congratulate you because you didn't do your drug of choice. You're still clean this long from your drug of choice. I've been 8 months clean off heroin. So, for me, as long as I don't use heroin, I am doing good.

A participant provided a notable response to the previous quote, stating, "Maybe we are all inherently hardwired to believe in harm reduction."

The negative case analysis revealed that only $1(3 \%)$ participant shared an experience that conflicted with the theme, and this conflicting statement was specific to the use of buprenorphine/naloxone and methadone. The participant shared:

I feel like Vivitrol would help because you can't get high if you have that, but in my personal opinion, I don't feel like you should take a medication [buprenorphine/naloxone or methadone] because it's just going from one drug to another, like using it as a crutch.

\section{Discussion}

Overall, drug court participants in this study felt that medications such as Suboxone (buprenorphine/naloxone), Vivitrol (naltrexone), and methadone were helpful in many ways, such as reducing cravings, minimizing withdrawal symptoms, and improving functioning in 
major areas of life, such as family relationships and employment. This belief was shared by both drug court participants who were currently taking MATs to support their recovery and those who were not on MATs.

As noted by participants in this study, a recommended approach to treating those who have opioid use disorders is to utilize MATs in combination with other psychosocial treatments. This theme was promising because it provided validation that using MATs in combination with psychosocial interventions is perceived by drug court participants as an effective approach for treating opioid use disorders, which aligns with the guidelines and recommendations of the American Society of Addiction Medicine for treating opioid use disorders (Kampman \& Jarvis, 2015). Since drug courts have not often supported the use of MATs and have traditionally created barriers to the use of these treatments, it was promising that participants still perceived these treatments to be helpful despite messages they might receive otherwise. For example, despite the evidence that MATs and counseling are effective interventions in treating opioid use disorders, many U.S. drug courts do not allow participants on opioid agonists and some judges or other stakeholders order participants to discontinue medications, even when they are recommended by a medical professional (Knopf, 2015). The magnitude of this problem has warranted intervention from the U.S. federal government, and the Substance Abuse and Mental Health Services Administration (SAMHSA) recently released a statement emphasizing that SAMHSA-funded drug courts can no longer deny individuals admission into drug court because they are on an opioid agonist or mandate individuals to discontinue the use of MATs (Knopf, 2015). Despite these barriers, the participants from this study provide validation that MATs are viewed as helpful in combination with psychosocial therapies, such as cognitive restructuring, motivational interviewing, and relapse prevention. Therefore, it is recommended that drug 
courts allow participants to take MATs and also refer them to treatment providers who have expertise in cognitive and behavioral therapies.

Participants' responses also highlighted a discrepancy between drug court expectations and the harm reduction approach used by many participants, and the way both parties defined and conceptualized harm reduction. For example, the drug court in this study acknowledges that the use of full (e.g. methadone) or partial (e.g. buprenorphine) opioid agonists to support recovery is consistent with a harm reduction model and permitted in the drug court when used under medical supervision. Some of the drug court participants in this study, however, reported another form of harm reduction that is not permitted in the drug court. After taking MATs, some participants reported that they discontinued opioid use but then began using other drugs. This would be consistent with a harm reduction approach to the treatment of substance use disorders, as the risk of overdose and death is greatly diminished if an individual is no longer using opioids.

U.S. drug courts will most likely not see this as an acceptable harm reduction approach to recovery, though, since most U.S. drug courts are guided by an abstinence-only philosophy of treatment. Reducing drug use or switching from more harmful drugs (e.g. opioids) to less harmful drugs (e.g. marijuana) are typically not seen as progress in U.S. drug courts. Continued drug use of any type could even result in progressive sanctions and ultimately termination from drug court. Whether a drug court operates under an abstinence-based or harm reduction lens of treatment and recovery, it is important for drug courts to be aware of the behavior identified in this study and, consistent with the drug court model (NADCP, 2004), respond in a nonadversarial, therapeutic manner.

The abstinence-only philosophy is not universal, however, particularly with non-U.S. drug courts (Holst, 2010). Irish drug treatment courts are one example (Loughran et al., 2015). 
If a drug court participant enters the Irish program using opioids and eliminates their opioid use throughout treatment, yet continues to use alcohol and/or marijuana, this may be viewed as progress and could still lead to program completion. In Ireland, the drug treatment court places tremendous value on assisting participants to develop skills for employment and enhancing their education. This is promising because employment and having at least a high school diploma or equivalent are consistently strong predictors of drug court completion and non-recidivism (Gallagher, 2013; Gill, 2016; Shaffer et al., 2011b). Drug court programming and polices vary from one country to the next, presumably to meet the individualized needs of the participants they serve.

\section{Limitations}

This study has several limitations. First, the findings of this study cannot be generalized beyond the research sample. The findings are limited to the 38 drug court participants who chose to participate in a focus group, and the themes drawn from the data are only applicable to the one drug court in this study. With that stated, however, it is plausible that the themes from this study, such as drug court participants practicing harm reduction, are applicable to other drug courts and the discussion may help guide drug court programming and future research. It is recommended that all drug courts incorporate qualitative methodologies into their program evaluations to assess their participants' experiences in drug court. Additionally, program evaluation is a key component of the drug court model (NADCP, 2004), and incorporating qualitative methodologies into program evaluations will capture data that quantitative methodologies simply cannot, such as the benefits of and barriers to using MATs in drug court.

Overall, MATs are an established and effective intervention for treating opioid use disorders. However, MATs have limitations and barriers to service delivery, particularly in the 
criminal justice system. The experiences and opinions of drug court stakeholders, who play a key role in the service delivery of MATs in drug court, are not captured in this study. As a result, it is recommended that future research collect data on drug court stakeholders' views on the use of MATs in the program. It is suspected that stakeholders, such as the judge, court coordinator, treatment providers, prosecutor, and defense attorneys, will offer a unique insight into the role of drug courts in treating opioid use disorders. Last, as common in qualitative research, the findings may have been impacted by social desirability bias. This is particularly true in this study, as data were collected through focus groups and research participants may have been selective in what they shared because other drug court participants were present. Focus groups have many strengths, such as offering synergistic and comprehensive answers to questions, but can also be impacted more heavily by social desirability bias. To potentially minimize this limitation, future research should consider collecting data through individual interviews. 


\section{References}

American College of Obstetricians and Gynecologists. ACOG Committee Opinion Number 711, August 2017. Washington, DC: Author. Retrieved from https://www.acog.org/ClinicalGuidance-and-Publications/Committee-Opinions/Committee-on-ObstetricPractice/Opioid-Use-and-Opioid-Use-Disorder-in-Pregnancy?IsMobileSet=false

Bronson, J., Stroop, J., Zimmer, S., \& Berzofsky, M. (2017). Drug Use, Dependence, and Abuse among State Prisoners and Jail Inmates, 2007-2009. US Department of Justice.

Brown, R. (2011). Drug court effectiveness: A matched cohort study in the Dane County drug treatment court. Journal of Offender Rehabilitation, 50, 191-201.

Center for Disease Control and Prevention. (2017). Annual surveillance report of drug-related risks and outcomes - United States, 2017. Retrieved from https://www.cdc.gov/drugoverdose/pdf/pubs/2017-cdc-drug-surveillance-report.pdf

Friedmann, P. D., Hoskinson Jr., R., Gordon, M., Schwartz, R., Kinlock, T., Knight, K., ...Frisman, L. K. (2012). Medication-assisted treatment in criminal justice agencies affiliated with the Criminal Justice-Drug Abuse Treatment Studies (CJ-DATS): Availability, barriers, and intentions. Substance Abuse, 33(1), 9-18.

Gallagher, J. R. (2013). Drug court graduation rates: Implications for policy advocacy and future research. Alcoholism Treatment Quarterly, 31, 241-253.

Gallagher, J. R., Nordberg, A., Deranek, M. S., Ivory, E., Carlton, J., \& Woodward Miller, J. (2015). Predicting termination from drug court and comparing recidivism patterns: Treating substance use disorders in criminal justice settings. Alcoholism Treatment Quarterly, 33(1), 28-43. 
Gallagher, J. R., \& Nordberg, A. (2017). A phenomenological and grounded theory study of women's experiences in drug court: Informing practice through a gendered lens. Women \& Criminal Justice, 27(5), 327-340.

Gallagher, J. R., \& Nordberg, A. (2018a). African American participants'suggestions for Eliminating racial disparities in graduation rates: Implications for drug court practice. Journal for Advancing Justice, 1, 89-107.

Gallagher, J. R., Wahler, E. A., Lefebvre, E., Paiano, T., Carlton, J., \& Woodward Miller, J. (2018b). Improving graduation rates in drug court through employment and schooling opportunities and medication-assisted treatment (MAT). Journal of Social Service Research, 44(3), 343-349.

Gallagher, J. R., \& Wahler, E. A. (2018c). Racial disparities in drug court graduation rates: The role of recovery support groups and environments. Journal of Social Work Practice in the Addictions, 18(2), 113-127.

Gill, M. E. (2016). Predictors of drug court client graduation. Journal of Offender Rehabilitation, $55(8), 564-588$.

Gottfredson, D. C., \& Exum, M. L. (2002). The Baltimore City drug treatment court: One-year results from a randomized study. Journal of Research in Crime and Delinquency, 39, 337-356.

Gottfredson, D. C., Najaka, S. S., \& Kearley, B. (2003). Effectiveness of drug treatment courts: Evidence from a randomized trial. Criminology \& Public Policy, 2, 171-196.

Holst, K. Y. W. (2010). A good score?: Examining twenty years of drug courts in the United States and abroad. Valparaiso University Law Review, 45(1), 73-106. 
Kampman, K. \& Jarvis, M. (2015). American Society of Addiction Medicine (ASAM) national practice guideline for the use of medications in the treatment of addiction involving opioid use. Journal of Addiction Medicine, 9(5), 358-367.

Knopf, A. (2015). SAMHSA-funded drug courts can’t order patients off meds. Alcoholism \& Drug Abuse Weekly, 27, 4-6.

Loughran, H., Hohman, M., Carolan, F., \& Bloomfield, D. (2015). Practice note: The Irish drug treatment court. Alcoholism Treatment Quarterly, 33(1), 82-92.

Matusow, H., Dickman, S. L., Rich, J. D., Fong, C., Dumont, D. M., Hardin, C., ...Rosenblum, A. (2013). Medication assisted treatment in US drug courts: Results from a nationwide survey of availability, barriers and attitudes. Journal of Substance Abuse Treatment, $44(5), 473-480$.

Mehta, C. \& Møllmann, M. (2017). Neither Justice nor Treatment: Drug Courts in the United States. Washington D.C.: Physicians for Human Rights.

Miles, M. B., Huberman, A. M., \& Saldana, J. (2014). Qualitative data analysis: A methods sourcebook ( $3^{\text {rd }}$ ed.). Thousand Oaks, CA: Sage Publications, Inc.

Mitchell, O., Wilson, D. B., Eggers, A., \& MacKenzie, D. L. (2012). Assessing the effectiveness of drug courts on recidivism: A meta-analytic review of traditional and non-traditional drug courts. Journal of Criminal Justice, 40, 60-71.

National Association of Drug Court Professionals [NADCP]. (2004). Defining drug courts: The key components. Retrieved from https://www.ncjrs.gov/pdffiles1/bja/205621.pdf

National Institute on Drug Abuse (2018). Effective Treatments for Opioid Addiction. Rockville, MD: Author. Retrieved from https://www.drugabuse.gov/publications/effective$\underline{\text { treatments-opioid-addiction/effective-treatments-opioid-addiction }}$ 
Padgett, D. K. (2016). Qualitative methods in social work research (3 ${ }^{\text {rd }}$ ed.). Thousand Oaks, CA: Sage Publications, Inc.

Rubin, A., \& Babbie, E. R. (2008). Research methods for social work (6 $6^{\text {th }}$ ed.). Belmont, CA: Thomson Brooks/Cole.

Rudd, R.A., Seth, P., David, F., Scholl, L. (2016). Increases in Drug and Opioid-Involved Overdose Deaths — United States, 2010-2015. Morbidity and Mortality Weekly Report, 65, Pgs.1445-1452.

Shaffer, D. K. (2011a). Looking inside the black box of drug courts: A meta-analytic review. Justice Quarterly, 28, 493-521.

Shaffer, D. K., Hartman, J. L., Listwan, S. J., Howell, T., \& Latessa, E. J. (2011b). Outcomes among drug court participants: Does drug of choice matter? International Journal of Offender Therapy and Comparative Criminology, 55, 155-174.

Substance Abuse and Mental Health Services Administration [SAMHSA]. (2018). Medications for opioid use disorder. Treatment Improvement Protocol (TIP) Series 63, Executive Summary. HHS Publication No. (SMA) 18-5063EXSUMM. Rockville, MD: Author.

The Council of Economic Advisors. (2017). The underestimated cost of the opioid crisis. Washington, DC: Executive Office of the President of the United States.

The Sentencing Project (2018). Trends in U.S. Corrections. Retrieved from http://www.sentencingproject.org/publications/trends-in-u-s-corrections/

U.S. Department of Health and Human Services [HHS]. (2017). Secretary Price announces HHS strategy for fighting opioid crisis. Retrieved from https://www.hhs.gov/about/leadership/secretary/speeches/2017-speeches/secretary-price$\underline{\text { announces-hhs-strategy-for-fighting-opioid-crisis/index.html }}$ 
U.S. Department of Health and Human Services [HHS]. (2018). What is the U.S. opioid epidemic? Retrieved from https://www.hhs.gov/opioids/about-the-epidemic/index.html U.S. Department of Justice, Office of Justice Programs. (2018). Drug courts. Retrieved from https://www.ncjrs.gov/pdffiles 1/nij/238527.pdf

Vandermause, R., Severtsen, B., \& Roll, J. (2013). Re-creating a vision of motherhood: Therapeutic drug court and the narrative. Qualitative Social Work, 12(5), 620-636.

Volkow, N. D. (2014). Testimony to congress: Prescription opioid and heroin abuse. Retrieved from https://www.drugabuse.gov/about-nida/legislative-activities/testimony-tocongress/2015/prescription-opioid-heroin-abuse 